Bull. Austral. Math. Soc.

VoL. 37 (1988) [107-111]

\title{
PROJECTIVE TENSOR PRODUCTS AND THE DUNFORD-PETTIS PROPERTY
}

\author{
Stephen P. Stehle
}

\begin{abstract}
We show the existence, in $c_{0} \otimes_{\gamma} c_{0}$, of a shrinking, subsymmetric basic sequence $\left(z_{i}\right)$ having no $c_{0}$ subsequences. In particular, the coefficient functionals for $\left(z_{i}\right)$ are weakly null and the subspace $\overline{s p}\left(z_{i}\right)$ fails to have the Dunford-Pettis property.
\end{abstract}

\section{Preliminaries}

Throughout, we will use $\lambda$ and $\gamma$ for the least and greatest crossnorms repsectively. The sequence spaces $c_{0}$ and $l_{1}$ will be given their usual meanings and norms. The unit vectors for these spaces will be given by $\left(e_{n}\right)$ and $\left(e_{n}^{*}\right)$. It is known that the sequences $\left(e_{i} \otimes e_{j}\right)$ and $\left(e_{i}^{*} \otimes e_{j}^{*}\right)$ are respective conditional bases for $c_{0} \otimes_{\gamma} c_{0}$ and $l_{1} \otimes_{\gamma} l_{1}$. The assumed ordering for both of these bases is the "upper rectangular" ordering given in [3]. A vector $x=\sum_{i j} \beta_{i j} e_{i}^{*} \otimes e_{j}^{*}$ in $l_{1} \otimes \lambda l_{1}$ will be written in matrix form $\left(\beta_{i j}\right)$ for short. Again, the sum is in the [3] ordering.

We recall that the definition of $\lambda(w)$ for $w=\left(\beta_{i j}\right)$ in $l_{1} \otimes_{\lambda} l_{1}$ is given by:

$$
\lambda(w)=\sup \sum_{i}\left|\sum_{j} \eta_{j} \beta_{i j}\right|=\sup \sum_{j}\left|\sum_{i} \zeta_{i} \beta_{i j}\right| .
$$

Here, the suprema are taken over $\left(\eta_{j}\right)$ and $\left(\zeta_{i}\right)$ in ball $l_{\infty}$.

The spaces $c_{0} \otimes \gamma c_{0}$ and $l_{1} \otimes_{\lambda} l_{1}$ are among many spaces identifiable as "matrix spaces". Such spaces are studied extensively in [5]. In that paper, the authors define spaces of infinite, finitely non-zero, matrices endowed with a variety of "matrix norms". The " $n$th main triangle projection" on the completion of such a space is given by:

$$
T_{n}\left(\left(a_{i j}\right)\right)=\sum_{i+j \leqslant n+1} a_{i j} u_{i j}
$$

where $u_{i j}$ has 1 in position $i j$; and 0 elsewhere.

Of particular interest to us are the matrix spaces $M_{\lambda_{11}}$ and $M_{\lambda_{11}^{*}}$ which are isometric to $l_{1} \otimes_{\lambda} l_{1}$ and $c_{0} \otimes_{\gamma} c_{0}$ respectively. (See [5], p. 45, for the definition of

Received 6 April 1987

The results in this paper first appeared in the author's Ph.D. dissertation written at lient State University under the direction of Professor J. Diestel.

Copyright Clearance Centre, Inc. Serial-fee code: 0004-9729/88 \$A2.00+0.00. 
conjugate norms $\alpha^{*}$; and p. 47 for the definition of the norms $\lambda_{p q}$ ). In Proposition 1.2 of [5], the authors show that:

$$
\left\|T_{n}\right\| \geqslant C \log (n)
$$

for some $C>0$ independent of $n$; where the underlying space is $M_{\lambda_{11}}$. Later, they show (Lemma 6.1, p. 64) that $\lambda_{11}^{*}\left(b_{n}\right)=\left\|T_{n}\right\|$ for the matrix $b_{n}$ defined by:

$$
b_{n}(i, j)= \begin{cases}1 & \text { for } n \geqslant i \geqslant j \geqslant 1 \\ 0 & \text { otherwise. }\end{cases}
$$

The result given in the abstract will be proved by a sequence of propositions, beginning with the fullowing duality result.

Proposition 1. The dual space of $c_{0} \otimes_{\gamma} c_{0}$ is identifiable with $l_{1} \otimes_{\lambda} l_{1}$. In particular, the basis $\left(e_{i} \otimes e_{j}\right)$ is shrinking.

Proof: It is well known [1] that $\left(c_{0} \otimes_{\gamma} c_{0}\right)^{*}$ is canonically identifiable with $L\left(c_{0}, l_{1}\right)$, the space of continuous linear operators from $c_{0}$ into $l_{1}$. The action of the identifying mapping is accomplished by extending the transformation:

$$
T(x \otimes y)=(T x)(y) \quad ; \text { for } T \in L\left(c_{0}, l_{1}\right) .
$$

It is easily established directly or by using [2] that each element of $L\left(c_{0}, l_{1}\right)$ is a weakly compact operator; hence compact, since $l_{1}$ has the Schur property. Thus, because $c_{0}^{*}=l_{1}$ has Grothendieck's approximation property, the algebraic tensor product $l_{1} \otimes l_{1}$ (under the injective norm) is canonically identifiable with a norm dense linear subspace of $L\left(c_{0}, l_{1}\right)[\mathbf{1}]$.

That $\left(e_{i} \otimes e_{j}\right)$ is shrinking follows from the established duality and the fact that $\left(e_{i}^{*} \otimes e_{j}^{*}\right)$ are the coefficient functionals.

We now define the basic sequence $\left(z_{i}\right) \subseteq c_{0} \otimes_{\gamma} c_{0}$ as follows:

$$
z_{i}=e_{i} \otimes \sum_{j=1}^{i} e_{j} .
$$

Since $\left(z_{i}\right)$ is a block sequence of a shrinking basis, it is shrinking as well. Also, it is clearly normalized. Moreover, by our duality result and the Hahn-Banach theorem, the norm in $\overline{s p}\left(z_{i}\right)$ is given by:

$$
\gamma\left(\sum_{i} \alpha_{i} z_{i}\right)=\sup \left|\sum_{i} \alpha_{i} w\left(z_{i}\right)\right| .
$$

Here, the $\alpha_{i}$ 's are scalars and the sup is over all $w=\left(\beta_{i j}\right) \in$ ball $l_{1} \otimes_{\lambda} l_{1}$.

Before our next result, recall that a basic sequence is called sub-symmetric if it is unconditional and equivalent to each of its subsequences. 
Proposition 2. The basic sequence $\left(z_{i}\right)$ is sub-symmetric.

Proof: We first show unconditionality. To do this, we let $N$ be a fixed integer, $I=\left\{i_{1}, i_{2}, \ldots, i_{k}\right\} \subseteq\{1,2, \ldots, N\}$, and $\left(\alpha_{i}\right)_{i \leqslant N}$ be arbitrary scalars. By the above remarks on the $\gamma$ norm, we can show that

$$
\gamma\left(\sum_{i_{p} \in I} \alpha_{i_{p}} z_{i_{p}}\right) \leqslant \gamma\left(\sum_{i \leqslant N} \alpha_{i} z_{i}\right)
$$

if given $w=\left(\beta_{i j}\right) \in$ ball $l_{1} \otimes_{\lambda} l_{1}$, there is a $\hat{w}$ in the same ball for which:

$$
\left|w\left(\sum_{i_{p} \in I} \alpha_{i_{p}} z_{i_{p}}\right)\right|=\left|\hat{w}\left(\sum_{i \leqslant N} \alpha_{i} z_{i}\right)\right| .
$$

So, let such a $w$ be given. Then from the definition of the $\lambda$ norm, the vector $\hat{w}=\left(\hat{\beta}_{i j}\right)$ formed from $w$ by replacing all rows in $\{1,2, \ldots, N\}-I$ with zeros is still in ball $l_{1} \otimes_{\lambda} l_{1}$ and satisfies the above equation. Thus $\left(z_{i}\right)$ is unconditional with unconditional constant $\leqslant 1$.

Next, we first show that for a given increasing sequence $\left(i_{k}\right)$ of integers, any fixed integer $K$, and scalars $\left(\alpha_{k}\right)_{k \leqslant K}$ that:

$$
\gamma\left(\sum_{k=1}^{K} \alpha_{k} z_{k}\right) \leqslant \gamma\left(\sum_{k=1}^{K} \alpha_{k} a_{i_{k}}\right)
$$

For this it suffices to show that given $w=\left(\beta_{i j}\right) \in$ ball $l_{1} \otimes_{\lambda} l_{1}$, there is $\hat{w}=\left(\hat{\beta}_{i j}\right)$ in the same ball satisfying the equations:

$$
w\left(z_{k}\right)=\hat{w}\left(z_{i_{k}}\right) ; 1 \leqslant k \leqslant K .
$$

This is easily done by "spreading out" the matrix for $w$ and defining $\hat{w}$ 's coordinates by

$$
\begin{aligned}
& \hat{\beta}_{i_{p} i_{q}}=\beta_{p q}, 1 \leqslant p, q \leqslant K \\
& \hat{\beta}_{i j}=0 \text { for all other positions. }
\end{aligned}
$$

Clearly, $\hat{w}$ satisfies the equations and, by the definition of the $\lambda$ norm, $\lambda(\hat{u})=\lambda(w)$. To get the other half of inequality $\left({ }^{*}\right)$ it suffices to solve the equations:

$$
w\left(z_{i_{k}}\right)=\hat{w}\left(z_{k}\right), \quad 1 \leqslant k \leqslant K
$$


where $\lambda(w) \leqslant 1$ is given and $\hat{w}$ satisfying $\lambda(\hat{w}) \leqslant 1$ is to be found. To get $\hat{w}=\left(\hat{\beta}_{p q}\right)$, we define

$$
\hat{\beta}_{p q}=\sum_{i_{q-1}+1 \leqslant t \leqslant i_{q}} \beta_{i_{p} t} ; \text { where } i_{0} \equiv 0
$$

It is straightforward that $\hat{w}$ satisfies the required equations. To see that $\lambda(\hat{w}) \leqslant 1$, suppose that $\left(\eta_{k}\right) \in$ ball $l_{\infty}$ is arbitrary. We define $\left(\xi_{j}\right) \in$ ball $l_{\infty}$ by: $\xi_{j}=\eta_{k}$ for $i_{k-1}+1 \leqslant j \leqslant i_{k}$. Then

$$
\sum_{i}\left|\sum_{k} \eta_{k} \hat{\beta}_{i k}\right|=\sum_{i}\left|\sum_{j} \xi_{j} \beta_{i j}\right| \leqslant 1 .
$$

Thus, by taking the supremum over $\left(\eta_{k}\right)$, we get $\lambda(\hat{w}) \leqslant 1$ as desired.

The following is the crucial step in our proof:

Proposition 3. The basic sequence $\left(z_{i}\right)$ has no $c_{0}$ subsequences.

Proof: This is shown in [5] (Lemma 6.1, p. 64). Specifically, the authors show the existence of an independent constant $C>0$ for which:

$$
\gamma\left(\sum_{i=1}^{n} z_{i}\right) \geqslant C \log (n) ; \text { for all } n
$$

Thus, $\left(z_{i}\right)$ is not a $c_{0}$ sequence; and, by Proposition 2, it has no $c_{0}$ subsequences.

Proposition 4. If $Z=\overline{s p}\left(z_{i}\right)$, then the coefficient functionals $\left(f_{i}\right) \subseteq Z^{*}$ are $\sigma\left(Z^{*}, Z^{* *}\right)$ null. In particular, $Z$ fails to have the Dunford-Pettis property.

Proof: Let $F \in Z^{* *}$ have norm equal to one. If $K$ is a fixed integer we choose $z \in$ ball $Z$ for which $\left|f_{i}(z)-F\left(f_{i}\right)\right| \leqslant 2^{-i}$ for $i \leqslant K$. The triangle inequality yields:

$$
\left\|\sum_{i=1}^{K} F\left(f_{i}\right) z_{i}\right\| \leqslant\|F\|+\|z\| \leqslant 2 .
$$

By the unconditionality of $\left(z_{i}\right)$ and the log inequality given above, the sequence $\left(F\left(f_{i}\right)\right)$ is an element of $c_{0}$; whence, $\left(f_{i}\right)$ is weakly null. Finally, since $\left(z_{i}\right)$ is shrinking, it is weakly null; so $Z$ fails the Dunford-Pettis property by Grothendieck's criterion [4].

\section{REFERENCES}

[1] J. Diestel and J. Uhl, Vector Measures 15, Math Surveys, 1977.

[2] N. Dunford and J.T. Schwartz, Linear Operators Part 1, Interscience 1958. 
[3] B.R. Gelbaum and J. Gil de la Madrid, 'Bases of Tensor Products of Banach Spaces', Pacific J. Math. 11 (1961), 1281-1286.

[4] A. Grothendieck, 'Sur les applications lineares faiblement compactes d'espaces du type $C(K)$ ', Canac. J. Math. 5 (1953), 129-173.

[5] S. Kwapien and A. Pelczynski, 'The main triangle projection in matrix spaces and its application', Studia Math 34 (1970), 43-68.

\author{
Mathematical Sciences \\ Akron Univ \\ Akron, Ohio 44325 \\ United States of America
}

